\title{
ELEMENTOS DE JUNÇÃO EM TABLETS: caracterização de adesivos e solventes
}

\author{
Luis Henrique Alves Cândido; Prof. Dr. \\ DEG/FA/UFRGS - LdSM/EE/UFRGS \\ candido@ufrgs.br
}

\author{
Alexandra Jacques Machado Xerxenevsky \\ Acadêmica design de produto/FA/UFRGS \\ alexandra.jmx@gmail.com \\ Bruna Togni de Oliveira \\ Acadêmica design de produto/FA/UFRGS \\ togni.bruna@gmail.com \\ Camila Civardi Rissato \\ Acadêmica design de produto/FA/UFRGS \\ camila.civardi@gmail.com
}

\begin{abstract}
Resumo: Atualmente, os computadores portáteis vêm ultrapassando o volume de vendas em relação aos computadores de mesa. Dentre os produtos portáteis, o tablet, objeto de estudo desse trabalho, é um dos equipamentos que mais crescem em volume de vendas no Brasil e em diversos países. Assim, fica evidente e lógico, para a indústria desse segmento, que o design deve focar seus esforços no desenvolvimento desses produtos. Dessa forma, um dos grandes desafios da área de design de produtos eletroeletrônicos é aliar desempenho funcional, estética e baixo peso. Para tanto, são usadas várias formas de fixação e, entre essas, encontram-se os adesivos. Esse material visa facilitar a montagem dos componentes em uma estrutura de espessura reduzida, mas que, no entanto, pode dificultar sua manutenção e reciclagem. Nesse sentido, o presente trabalho, através da desmontagem de dois tablets, caracterizou com auxílio da técnica de FTIR, nove tipos de diferentes adesivos, que foram encontrados nos componentes internos desses produtos. A partir disso, foram selecionados dez tipos de solventes para a extração dos adesivos. A seleção teve como base de pesquisa a ficha técnica dos respectivos adesivos. Com essas informações, busca-se balizar o uso de soluções que venham incentivar a redução contínua do número de diferentes adesivos no mesmo produto e, consequentemente, reduzir o número de diferentes solventes aplicados para sua extração.
\end{abstract}

Palavras-chave: reciclagem, eletroeletrônico, tablet, adesivo, ecodesign. 


\begin{abstract}
Currently, laptops come surpassing the volume of sales compared to desktop computers. Among portable products, the tablet, object of study of this paper, is one of the fastest growing equipment by sales volume in Brazil and many other countries. That way, it is clear for this industry segment that the design should focus its efforts on the development of these products. Thus, a major challenge in the field of design of electronic products is to combine the excellent functional performance to the aesthetics and the low weight. For this, various forms of fixation are used and, among these, there are the adhesives. This material aims to facilitate the assembly of components on a structure reduced in thickness; however, it may impede maintenance and recycling. In this sense, the present work, by dismantling two tablets, characterized, with the support of the FTIR technique, nine kinds of different adhesives, which were found in the internal components of these products. Ten kinds of solvents for the extraction of the adhesives were selected. This selection was based on the technical file of the adhesives. With this information, we seek to guide the use of solutions that will encourage the reduction of the number of different adhesives on the same product and, consequently, reduce the number of solvents applied for the extraction.
\end{abstract}

Keywords: recycling, electronic, tablet, adhesive, ecodesign.

\title{
1. INTRODUÇÃO
}

Nas últimas décadas, a indústria dos eletrônicos tem revolucionado o comportamento mundial: os equipamentos eletrônicos tornaram-se essenciais para a vida moderna nos países industrializados e pós-industrializados. A indústria, com forte apoio da mídia, cria constantemente uma ilusão de obsolescência, lançando incessantemente no mercado novos aparelhos com inovação incremental. Essa indústria também trabalha com o estímulo ao consumo, que provoca comportamentos condicionados e gera o conceito de objeto de desejo. Os equipamentos já são produzidos e adquiridos com a certeza de que logo serão substituídos devido à introdução de novas tecnologias ou à indisponibilidade de peças de reposição. Esse esquema econômico é conhecido como "obsolescência programada" e vem assolando essa geração com, aproximadamente, 40 milhões de toneladas de e-lixo descartadas anualmente no mundo (SCHLUEP et al, 2009).

Segundo a Agência Brasileira de Desenvolvimento Industrial - ABDI (2012), no fim da sua vida útil, quando, teoricamente, todas as possibilidades de reparo, atualização ou reuso são esgotadas, esses aparelhos passam a ser considerados Resíduos de Equipamentos Eletroeletrônicos (REEE). Tendo como base o descarte de equipamentos com tecnologia mais recente, a relação entre consumo e descarte desses aparelhos toma proporções ainda mais alarmantes, como é o caso dos tablets e dos notebooks.

Visando apontar caminhos para a adequada reciclagem desses produtos, em agosto de 2010, foi promulgada a Lei 12.305, instituindo a Política Nacional de Resíduos Sólidos (PNRS). De acordo com o Compromisso Empresarial para Reciclagem 
- CEMPRE (2010), a PNRS reúne o conjunto de princípios, objetivos, instrumentos, diretrizes, metas e ações adotadas pelo Governo Federal visando à gestão integrada e ao gerenciamento ambientalmente adequado dos resíduos sólidos e, entre esses, encontra-se os eletroeletrônicos.

A regulamentação dessa lei foi feita pelo Decreto $n=7.404$, que regulariza as inovações introduzidas na gestão e gerenciamento dos resíduos sólidos pela PNRS, sendo a principal delas o sistema de logística reversa. Esse sistema tem como intuito recondicionar os resíduos sólidos ao setor empresarial para reaproveitamento no ciclo produtivo ou para destinação final ambientalmente adequada (CEMPRE, 2010).

Uma das questões que atrasam e impedem a prática da logística reversa é o alto custo das técnicas de reciclagem do e-lixo, uma vez que existe maior investimento em tecnologias de reprocessamento desse lixo em países mais desenvolvidos. Outro fator que prejudica a reciclagem de certos componentes é a necessidade da miniaturização dos elementos internos dos produtos. Fica evidente que os principais fabricantes de tablets são líderes no desenvolvimento de componentes para seus produtos (SCHLUEP et al, 2009). Dessa forma, a miniaturização tende a ficar restrita às empresas líderes, entretanto, para que a redução de peso seja observada e sentida pelo usuário, os sistemas de montagem devem proporcionar arranjos eficientes de montagem.

Ao buscar esses arranjos, muitas vezes a área de projeto precisa utilizar processos de montagem eficientes, mas que podem dificultar a desmontagem, tanto para manutenção como para reciclagem. Dentre esses processos, a utilização de adesivos é uma técnica bastante utilizada para montagem, visto que, ocupa um espaço reduzido e auxilia no amortecimento e vedação. Porém, segundo os preceitos do ecodesign (CÂNDIDO, 2008) deve-se buscar reduzir o uso de adesivos, isso porque, esse elemento de junção pode prejudicar, principalmente, a reciclagem dos componentes. Nesse sentido, para que ocorra uma reflexão sobre a utilização e redução do uso de multiadesivos, o presente trabalho apresenta a correlação entre esse elemento e o solvente indicado para sua extração.

\section{O TABLET}

O registro da primeira patente de um sistema com capacidade para reconhecer a escrita por meio de movimentos da mão ocorreu em 1915 e a primeira demonstração de reconhecimento de escrita manual sem a utilização de um teclado convencional se deu em 1956. É nos anos 60, no entanto, que surge o conceito de Tablet PC, quando Alan Kay e a Xerox Palo Alto Research Center produziram o Dynabook (Figura 1). O objetivo desse dispositivo era auxiliar e facilitar a educação de crianças (SANTANA, s.d.).

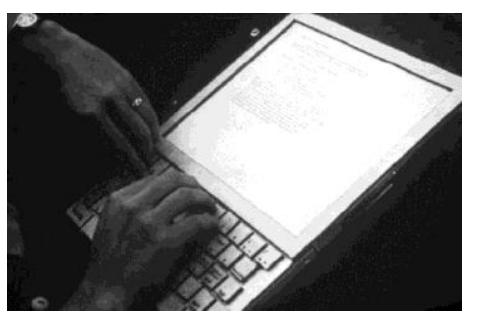

Figura 1 - 0 Dynabook.

Fonte: Edible Apple, 2010. 
Somente no fim dos anos 80 e início dos 90 que surgem os primeiros aparelhos semelhantes aos tablets atuais. Nos anos seguintes, foram lançados tablets diversos, como o Newton MessagePad da Apple, o Windows XP Tablet Edition, da Microsoft, o Compaq TC1000 e o AmazonKindle. Nenhum desses, porém, obteve o impacto desejado no mercado de vendas. Foi com o lançamento do iPad da Apple, em 2010, que as vendas de tablet rapidamente se expandiram, iniciando a explosão rentável desses aparelhos. A partir de então, todas as outras marcas se viram desafiadas a superar suas características iniciais, como o peso, a autonomia, a definição do ecrã, a qualidade do áudio e da câmera, as aplicações disponíveis e o design, influenciando diretamente na decisão final do consumidor (SANTANA, s.d.).

Dados apontados pela Associação Brasileira da Indústria Elétrica e Eletrônica ABINEE (2014), mostram que o Brasil é o maior produtor per capita de resíduos de computadores pessoais entre os países emergentes $(0,5 \mathrm{~kg} / \mathrm{cap}$.ano). Isso se deve não somente ao grande consumo (Tabela 1), como também pela produção, reaproveitamento e reciclagem de aparelhos eletroeletrônicos.

Tabela 1 - Relação de consumo de desktops, notebooks e tablets por ano no Brasil.

\begin{tabular}{cccc}
$\begin{array}{c}\text { Mercado de PCs e } \\
\text { tablets } \\
\text { (em mil unidades) }\end{array}$ & Desktops & Notebooks & Tablets \\
\hline 2011 & 7.500 & 8.354 & 1.144 \\
\hline 2012 & 6.582 & 8.932 & 3.267 \\
\hline 2013 & 5.740 & 8.205 & 8.386 \\
\hline 2014 & 4.499 & 8.368 & 11.144 \\
\hline
\end{tabular}

Fonte: Adaptada da Associação Brasileira da Indústria Elétrica e Eletrônica - ABINEE.

Ao analisar esses dados, podemos apontar para o aumento da produção desses produtos e da necessidade contínua do desenvolvimento de pesquisas que auxiliem a área de design de produtos na aplicação de processos eficientes de montagem e de reciclagem.

\section{MATERIAIS E MÉTODOS}

Com o objetivo de analisar estruturalmente o tablet (modelo de 7" - diferentes fabricantes), foram desmontados dois desses produtos (Figura 2). A desmontagem foi realizada manualmente com o auxílio de chaves de precisão e chaves de fenda.

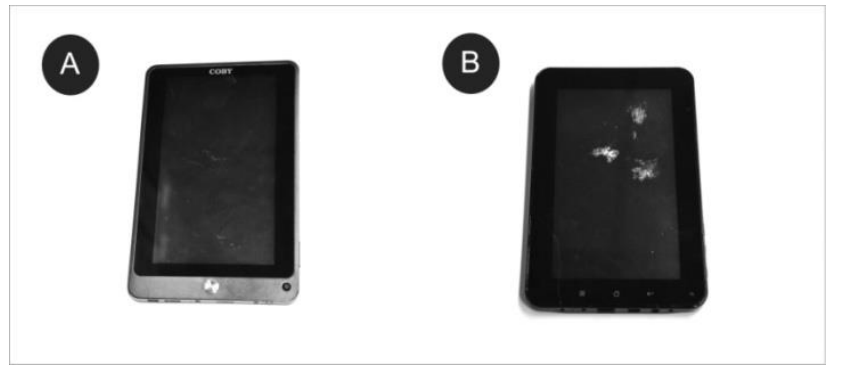

Figura 2 - Tablets analisados.

Fonte: Elaborado pelos autores, com base no ensaio realizado. 
Durante a desmontagem foram encontradas diversas áreas com resíduos de adesivos, em ambos os equipamentos, que após serem extraídas por raspagem, com uso de um bisturi, foram caracterizados via técnica do FTIR (Figura 3).

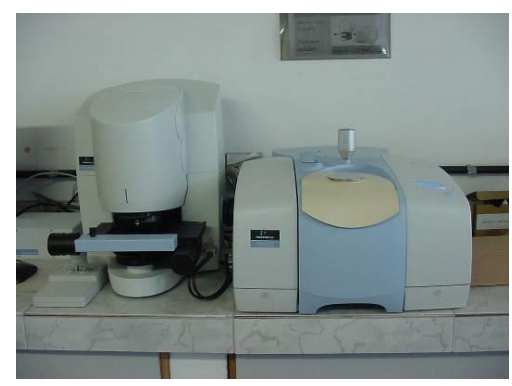

Figura 3 - Equipamento de FTIR (Local: LdSM/UFRGS).

Fonte: Elaborado pelos autores, com base no ensaio realizado.

Os espectros foram obtidos em espectrômetro (FTIR) marca PerkinElmer Spectrum 100 (Figura 3) com resolução de $4 \mathrm{~cm}-1$ realizando 16 varreduras por amostra na região de 4000 a $650 \mathrm{~cm}-1$.

\subsection{Estudo de modelo - Tablet A}

O modelo A (Figura 2) é representado por meio de sua estrutura funcional na Figura 4, 5 e 6, sendo os componentes identificados na Tabela 2. As letras na cor vermelha indicam os locais com uso de adesivos.
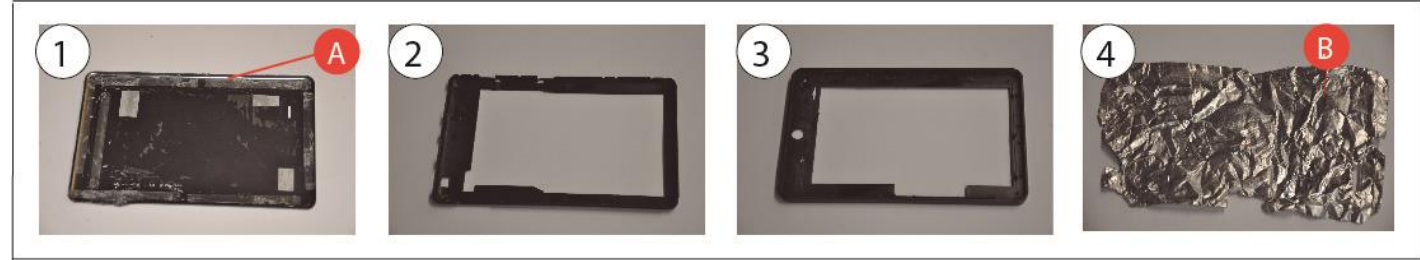

Figura 4 - Componentes estruturais externos do modelo A.

Fonte: Elaborado pelos autores.

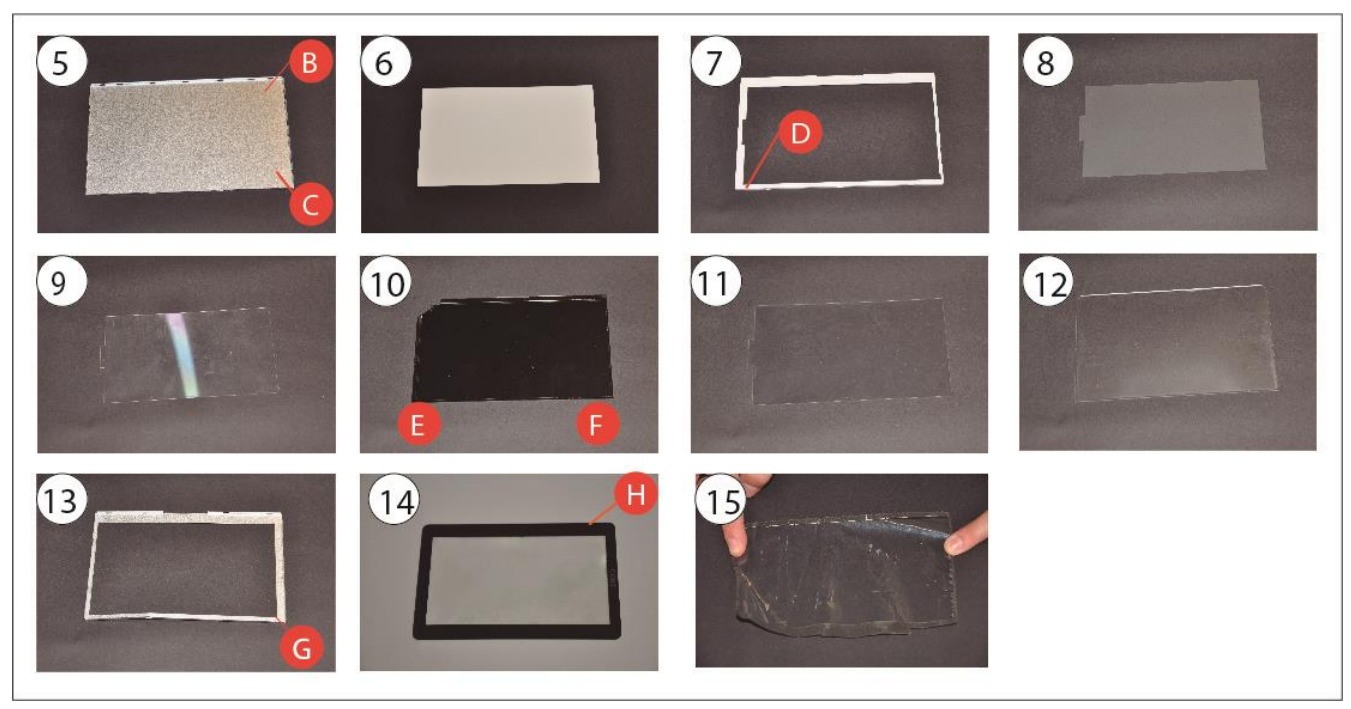

Figura 5 - Componentes da tela do modelo A.

Fonte: Elaborado pelos autores. 

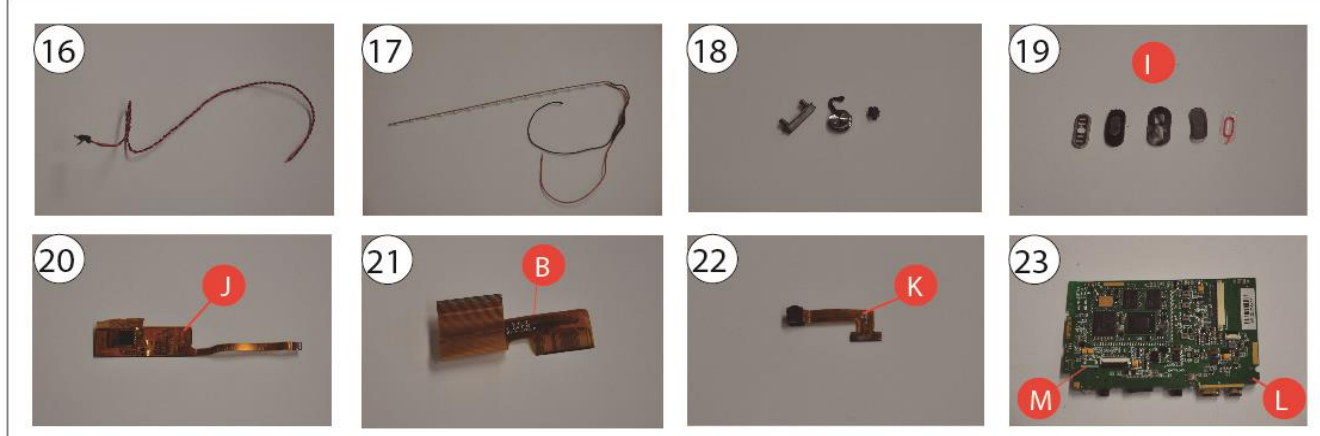

Figura 6 - Demais componentes presentes no modelo A.

Fonte: Elaborado pelos autores.

Como mostram as figuras, existem quinze pontos de aplicação de adesivos.

Tabela 2 - Identificação dos componentes do tablet A.

\begin{tabular}{|c|c|c|c|}
\hline Peça & Denominação & Material & Quantidade \\
\hline 1 & Base inferior & Alumínio & 1 \\
\hline 2 & Base interna & Polímero & 1 \\
\hline 3 & Base superior & Polímero & 1 \\
\hline 4 & Lâmina adesiva & Alumínio & 1 \\
\hline 5 & Fechamento do perfil 1 & Alumínio & 1 \\
\hline 6 & Lâmina 1 & Polímero & 1 \\
\hline 7 & Perfil 1 & Polímero & 1 \\
\hline 8 & Lâmina 2 & Polímero & 1 \\
\hline 9 & Lâmina 3 & Polímero & 1 \\
\hline 10 & Conjunto da tela & Multimateriais & 1 \\
\hline 11 & Lâmina 4 & Polímero & 1 \\
\hline 12 & Lâmina 5 & Polímero & 1 \\
\hline 13 & Perfil 2 & Alumínio & 1 \\
\hline 14 & Tela de toque & Polímero & 1 \\
\hline 15 & Lâmina da tela de toque & Polímero & 1 \\
\hline 16 & Fios & Multimateriais & 2 \\
\hline 17 & Componente de iluminação da tela (LED) & Multimateriais & 1 \\
\hline 18 & Botões & Multimateriais & 3 \\
\hline 19 & Componentes do alto-falante & Multimateriais & 5 \\
\hline 20 & Conexão 1 & Multimateriais & 1 \\
\hline 21 & Conexão 2 & Multimateriais & 1 \\
\hline 22 & Câmera e conexão & Multimateriais & 1 \\
\hline
\end{tabular}




\begin{tabular}{llll}
\hline 23 & Placa de circuito impresso & Multimateriais & 1 \\
\hline 24 & Parafusos & Aço & 3 \\
\hline & Total de componentes desmontados & & 33 \\
\hline
\end{tabular}

Fonte: Elaborado pelos autores.

\subsection{Estudo de modelo - Tablet B}

O modelo B (Figura 2) é representado por meio de sua estrutura funcional nas Figuras 7, 8 e 9, sendo os componentes identificados na Tabela 3. As letras na cor verde indicam os locais com uso de adesivos.

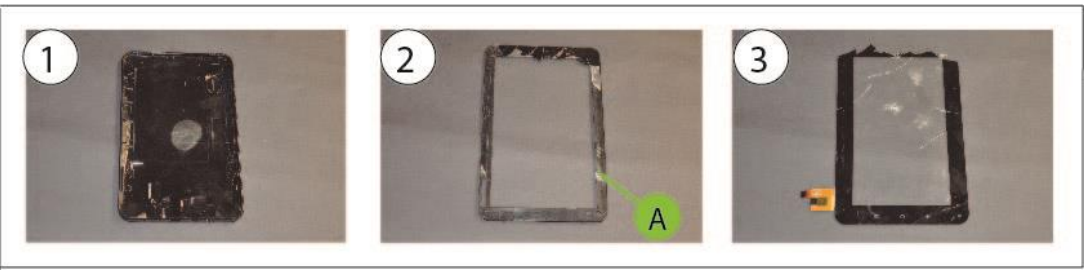

Figura 7 - Componentes estruturais externos do modelo B.

Fonte: Elaborado pelos autores.
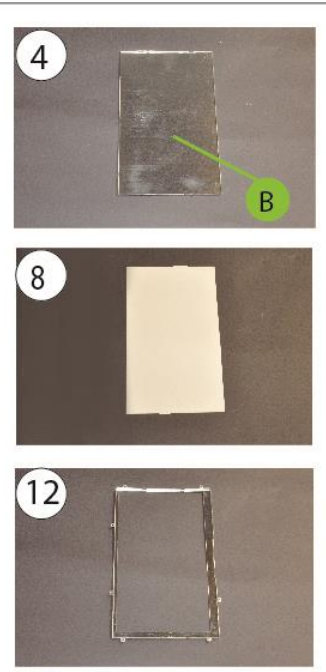
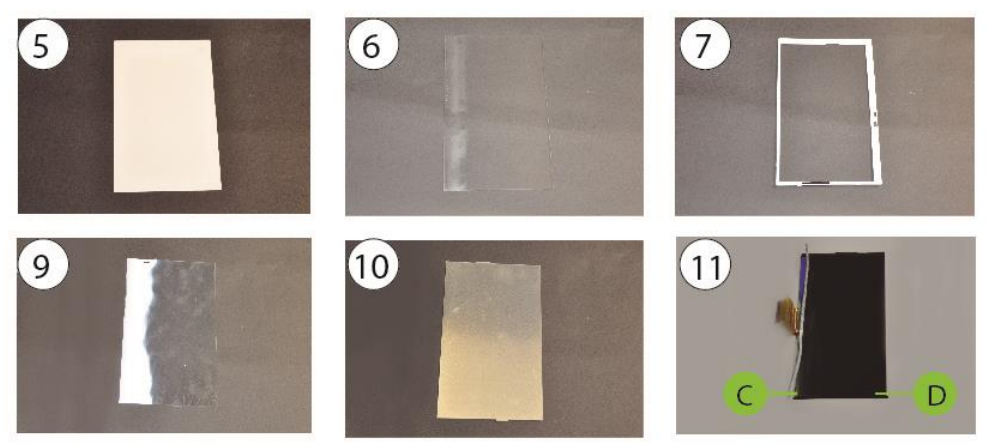

Figura 8 - Componentes da tela do modelo B.

Fonte: Elaborado pelos autores.
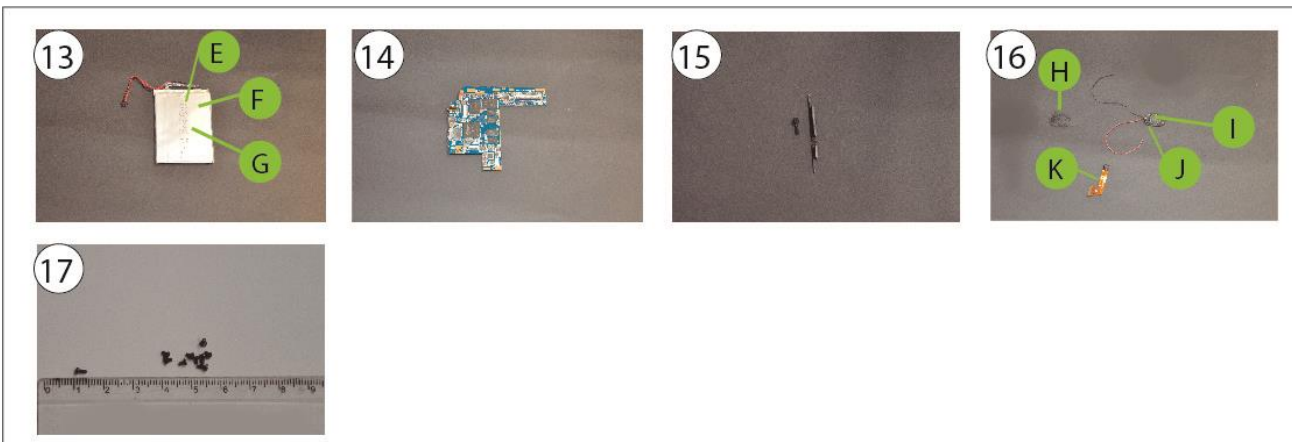

Figura 9 - Demais componentes presentes no modelo B.

Fonte: Elaborado pelos autores. 
Como mostram as figuras, existem onze pontos de aplicação de adesivos.

Tabela 3 - Identificação dos componentes do tablet B.

\begin{tabular}{|c|c|c|c|}
\hline Peça & Denominação & Material & Quantidade \\
\hline 1 & Base inferior & Polímero & 1 \\
\hline 2 & Base superior & Polímero & 1 \\
\hline 3 & Tela de toque & Multimateriais & 1 \\
\hline 4 & Fechamento do perfil 1 & Alumínio & 1 \\
\hline 5 & Lâmina 1 & Polímero & 1 \\
\hline 6 & Lâmina 2 & Polímero & 1 \\
\hline 7 & Perfil 2 & Polímero & 1 \\
\hline 8 & Lâmina 3 & Polímero & 1 \\
\hline 9 & Lâmina 4 & Polímero & 1 \\
\hline 10 & Lâmina 5 & Polímero & 1 \\
\hline 11 & Conjunto da tela & Multimateriais & 1 \\
\hline 12 & Perfil 1 & Alumínio & 1 \\
\hline 13 & Bateria & Multimateriais & 1 \\
\hline 14 & Placa de circuito impresso & Multimateriais & 1 \\
\hline 15 & Botões & Polímeros & 2 \\
\hline 16 & Componentes de áudio e vídeo & Multimateriais & 3 \\
\hline \multirow[t]{2}{*}{17} & Parafusos & Aço & 10 \\
\hline & Total de componentes desmontados & & 29 \\
\hline
\end{tabular}

Fonte: Elaborado pelos autores.

Ao comparar a Tabela 2 com a Tabela 3, observa-se que o número de componentes total fica muito próximo, mesmo em tablets de marcas distintas. Esse detalhe pode apontar para uma similarização de projetos, o que vem ao encontro do número restrito de fabricantes dos componentes internos. Ao analisar esses produtos, fica claro que, se por um lado o uso de adesivos facilita a montagem e auxilia na conservação da estrutura tornando-a mais resistente à queda, por outro, reflete a dificuldade na separação dos componentes e, consequentemente, na manutenção e possibilidade de reciclagem. Entre os modelos examinados, o equipamento $A$ apresentava maior quantidade de cola, o que repercutiu no seu tempo de desmontagem de 22 minutos contra os 17 minutos gastos para desmontagem do aparelho B.

\subsection{Caracterização dos adesivos}

Após a desmontagem dos tablets, os componentes (com adesivos) foram 
raspados, visando à extração do adesivo para análise via FTIR. Assim, na Tabela 4 e na Tabela 5, são apresentados os tipos de adesivos encontrados em cada componente, os fabricantes desses adesivos e os possíveis solventes a serem empregados para sua remoção. A busca pelo fabricante do adesivo foi executada a partir da biblioteca de materiais do próprio FTIR. De posse dessa informação, foi verificada a ficha técnica do adesivo e os possíveis solventes.

\subsubsection{Tablet modelo A}

A tabela abaixo mostra as informações referentes aos componentes do tablet A.

Tabela 4 - Identificação dos adesivos presentes nas peças e seus solventes.

\begin{tabular}{|c|c|c|c|c|}
\hline Peça & Tipo de Adesivo & Fabricante & Solventes 1 & Solventes 2 \\
\hline$A$ & Ektar PCGT 5445 & Eastman & $\begin{array}{c}\text { Trichloroacetaldehyde } \\
\text { hydrate e } \\
\text { chlorophenol }\end{array}$ & $\begin{array}{l}\text { Acetone e methyl } \\
\text { ethyl ketone }\end{array}$ \\
\hline B & TRI-FOIL 903 & $\begin{array}{l}\text { Saint Gobain } \\
\text { Performance } \\
\text { Products } \\
\end{array}$ & Não fornecido & Não fornecido \\
\hline $\mathrm{C}$ & $\begin{array}{l}\text { Poly (2-Ethylhexyl } \\
\text { acrylate) }\end{array}$ & Sigma-Aldrich & Ketone & Não fornecido \\
\hline $\mathrm{D}$ & ConforFoam C-47 & E-A-R & Toluene e isooctane & $\begin{array}{c}\text { Acetone e methyl } \\
\text { ethyl ketone }\end{array}$ \\
\hline$E$ & $\begin{array}{c}\text { Poly (2-Ethylhexyl } \\
\text { acrylate) }\end{array}$ & Sigma-Aldrich & Ketone & Não fornecido \\
\hline $\mathrm{F}$ & $\begin{array}{l}\text { Poly (2-Ethylhexyl } \\
\text { acrylate) }\end{array}$ & Sigma-Aldrich & Ketone & Não fornecido \\
\hline G & $\begin{array}{c}\text { Poly (2-Ethylhexyl } \\
\text { acrylate) }\end{array}$ & Sigma-Aldrich & Ketone & Não fornecido \\
\hline $\mathrm{H}$ & Rynite 555 & $\begin{array}{l}\text { DuPont Performance } \\
\text { Polymers }\end{array}$ & $\begin{array}{c}\text { Trichloroacetaldehyde } \\
\text { hydrate e } \\
\text { chlorophenol }\end{array}$ & $\begin{array}{l}\text { Acetone e methyl } \\
\text { ethyl ketone }\end{array}$ \\
\hline 1 & $\begin{array}{c}\text { Poly (2-Ethylhexyl } \\
\text { acrylate) }\end{array}$ & Sigma-Aldrich & Ketone & Não fornecido \\
\hline $\mathrm{J}$ & $\begin{array}{l}\text { Poly (2-Ethylhexyl } \\
\text { acrylate) }\end{array}$ & Sigma-Aldrich & Ketone & Não fornecido \\
\hline K & $\begin{array}{c}\text { Poly } \\
\text { (HexadecylAcrylate) }\end{array}$ & Sigma-Aldrich & Methanol e Heptane & Não fornecido \\
\hline $\mathrm{L}$ & $\begin{array}{c}\text { Poly (2-Ethylhexyl } \\
\text { acrylate) }\end{array}$ & Sigma-Aldrich & Ketone & Não fornecido \\
\hline M & Cyanacryl 35 & $\begin{array}{c}\text { American } \\
\text { CyanamidCompany }\end{array}$ & Não fornecido & Não fornecido \\
\hline
\end{tabular}

Fonte: Elaborado pelos autores.

\subsubsection{Tablet modelo B}

A tabela abaixo mostra as informações referentes aos componentes do tablet B. 
Tabela $\mathbf{5}$ - Identificação dos adesivos presentes nas peças e seus solventes.

\begin{tabular}{|c|c|c|c|c|}
\hline Peça & Tipo de Adesivo & Fabricante & Solventes 1 & Solventes 2 \\
\hline$A$ & Cyanacryl 35 & $\begin{array}{c}\text { American } \\
\text { CyanamidCompany }\end{array}$ & Não fornecido & Não fornecido \\
\hline B & $\begin{array}{c}\text { Poly(2-Ethylhexyl } \\
\text { acrylate) }\end{array}$ & Sigma-Aldrich & Ketone & Não fornecido \\
\hline$C$ & $\begin{array}{l}\text { Poly(2-Ethylhexyl } \\
\text { acrylate) }\end{array}$ & Sigma-Aldrich & Ketone & Não fornecido \\
\hline D & ConforFoam C-47 & $E-A-R$ & Toluene e isooctane & $\begin{array}{c}\text { Acetone e methyl } \\
\text { ethyl ketone }\end{array}$ \\
\hline $\mathrm{E}$ & Cyanacryl 35 & $\begin{array}{c}\text { American } \\
\text { CyanamidCompany }\end{array}$ & Não fornecido & Não fornecido \\
\hline$F$ & TRI-FOIL 903 & $\begin{array}{c}\text { Saint Gobain } \\
\text { Performance } \\
\text { Products }\end{array}$ & Não fornecido & Não fornecido \\
\hline G & SuperNevtac 99 & $\begin{array}{c}\text { Neville } \\
\text { ChemicalCompany }\end{array}$ & $\begin{array}{c}\text { Methanol, } \\
\text { isopropanol e } \\
\text { detergente }\end{array}$ & Não fornecido \\
\hline $\mathrm{H}$ & ConforFoam C-47 & $E-A-R$ & Toluene e isooctane & $\begin{array}{c}\text { Acetone e methyl } \\
\text { ethyl ketone }\end{array}$ \\
\hline 1 & ConforFoam C-47 & $E-A-R$ & Toluene e isooctane & $\begin{array}{c}\text { Acetone e methyl } \\
\text { ethyl ketone }\end{array}$ \\
\hline J & Cyanacryl 35 & $\begin{array}{c}\text { American } \\
\text { CyanamidCompany }\end{array}$ & Não fornecido & Não fornecido \\
\hline K & Petrothene NA 143 & EquistarChemicals & $\begin{array}{l}\text { Acetone e methyl } \\
\text { ethyl ketone }\end{array}$ & Não fornecido \\
\hline
\end{tabular}

Fonte: Elaborado pelos autores.

Ao avaliar as Tabelas 4 e 5, podemos apontar para uma diversidade de tipos de adesivos que chega a nove diferentes marcas e dez tipos de solventes, entre eles, o Trichloroacetaldehydehydrate, chlorophenol, Toluene, isooctane, Methanol, isopropanol,Acetone, methylethylketone, Heptanee o detergente de uso doméstico.

Destaca-se que para seis adesivos não foram encontrados na ficha técnica do fabricante os solventes compatíveis. Esse fato demonstra a grande dificuldade da busca de informações técnicas que auxiliem a área de design de produtos na seleção da melhor alternativa do tipo de adesivo a ser utilizado e o seu respectivo solvente.

\section{CONCLUSÃO}

Diante dos avanços tecnológicos aplicados aos tablets, principalmente na busca de uma espessura reduzida e leveza, o uso de adesivos vem tornando-se o principal elemento de fixação de seus componentes internos. No entanto, esse artifício de junção prejudica tanto o processo de separação dos componentes, quanto o reprocessamento dos polímeros, prejudicando, como, por exemplo, futuros processos de extrusão do material reciclado, devido aos resíduos de adesivos dispostos na superfície dos componentes.

Nesse sentido, o presente trabalho, ao relacionar o tipo de adesivo com seu respectivo solvente, busca incentivar a área de design de produtos na reflexão da problemática ambiental que esses dois materiais podem acarretar no ambiente. 
Dessa forma, esse trabalho visa balizar o uso de soluções que venham a reduzir essa problemática, como por exemplo, incentivar a redução contínua do número de diferentes adesivos no mesmo produto e, consequentemente, reduzir o número de solventes aplicados para sua extração.

\section{REFERÊNCIAS}

AGÊNCIA BRASILEIRA DE DESENVOLVIMENTO INDUSTRIAL. Logística reversa de equipamentos eletroeletrônicos: análise de viabilidade técnica e econômica. Brasília, 2012. $178 \mathrm{p}$.

ANDRADY, A. L. Plastics and the Environment. Hoboken: John Wiley \& Sons, 2003. 762 p.

ASSOCIAÇÃO BRASILEIRA DA INDÚSTRIA ELÉTRICA E ELETRÔNICA. Desempenho Setorial. Disponível em: <http://www.abinee.org.br/abinee/decon/decon15.htm>. Acesso em 20 mar. 2014.

BALBINOTTI, R. R.; PEREIRA, C. A.; SILVA, E.; KINDLEIN, W. J.; Princípios básicos de junção utilizados em sistemas e subsistemas de produtos industriais e sua importância no desenvolvimento sustentável. In: ANPPAS - Associação Nacional de Pós-Graduação e Pesquisa em Ambiente e Sociedade. Campinas - Sp. 2002.

BBC Brasil. Países pobres são destino de $\mathbf{8 0 \%}$ do lixo eletrônico de nações ricas. Disponível em: <http://www.bbc.co.uk/portuguese/noticias/2013/01/130118_lixo_eletronico_bg.sht $\mathrm{ml}$. Acesso em 10 jan. 2014.

BOOTHROYD, G; ALTING, L. Design for Assembly and Disassembly. CIRPAnnals Manufacturing Technology. v. 41, n. 2, 1992, p. 625-636.

CÂNDIDO, L. H. A. Contribuição ao estudo da reutilização, redução e da reciclagem dos materiais com aplicação do ecodesign. 2008. 130 p. Dissertação (Mestrado em Engenharia, ênfase: Ciência e Tecnologia dos Materiais) - Escola de Engenharia. Universidade Federal do Rio Grande do Sul, Porto Alegre. 2008.

COMPROMISSO EMPRESARIAL PARA RECICLAGEM. Política Nacional de Resíduos Sólidos: agora é lei. São Paulo, 2010. 5 p.

FERREIRA, D. C.; BEZERRA DA SILVA, J.; GALDINO, J. C. S. Reciclagem do e-lixo (ou lixo eletro-eletrônico). Disponível em: <http://observatorioderesiduos.com.br/wpcontent/uploads/2011/03/597.pdf>. Acesso em: 10 jan. 2014.

From Alan Kay's Dynabook to the Apple iPad. Disponível em: <http://www.edibleapple.com/2010/04/30/from-alan-kays-dynabook-to-the-appleipad/>. Acesso em: 10 abr. 2014. 
GUARNIERI, P. Logística Reversa: em busca do equilíbrio econômico e ambiental. Recife: Ed. Clube de Autores, 2011. 311 p.

MANZINI, E.; VEZZOLI, C. O Desenvolvimento de Produtos Sustentáveis: Os requisitos ambientais dos produtos industriais. São Paulo. EDUSP, 2002. 366 p.

MARQUES, A. C. Análise de Similares: Contribuição ao desenvolvimento de uma metodologia de seleção de materiais e ecodesign. 2008. 127 p. Dissertação (Mestrado em Engenharia) - Escola de Engenharia. Universidade Federal do Rio Grande do Sul, Porto Alegre. 2008.

OLIVEIRA, C. R. Alternativas Tecnológicas para tratamento e reciclagem do lixo de informática. Trabalho de conclusão de curso apresentado junto à atividade de ensino Projeto Tecnológico como requisito parcial para obtenção de grau - Instituto de Química. Universidade Federal do Rio Grande do Sul, Porto Alegre. 2010.

ROGERS, D. S.; TIBBEN-LEMBKE, R. S. GoingBackwards: Reverse LogisticsTrendsandPractices.Pittsburgh: Reverse LogisticsExecutiveCouncil, 1999. 280 p.

SANTANA, P. A história dos tablets: viagem no tempo. Disponível em: <http://www.mundodostablets.com.br/artigos/a-historia-dos-tablets-viagem-otempo/>. Acessoem: 10 abr. 2014.

SCHLUEP, M. et al. Recyclinf: from e-waste to resources. Berlin: Oktoberdruck AG, 2009. 120 p.

YAMANE, L. H. Recuperação de metais de placas de circuito impresso de computadores obsoletos através de processo biohidrometalúrgico. 2012. 128 p. Tese (Doutorado em Engenharia Metalúrgica e de Materiais) - Escola Politécnica da USP. Universidade de São Paulo, São Paulo. 2012. 